\title{
Hepatoprotective Role of $\alpha$-Lipoic acid and Thymoquinone in Acetaminophen- Induced Liver Injury: Down-Regulation of COX-2 and flt-1 Expression
}

\author{
Nawal M. Al-Rasheed ${ }^{1,2}$, Laila Fadda ${ }^{1}$, Nouf M. Al-Rasheed ${ }^{1}$, Iman H. Hasan ${ }^{1}$, Hanaa M. \\ $\mathrm{Ali}^{3,4^{*}}$, Musaed Al-Fayez ${ }^{5}$, Raeesa A. Mohamad ${ }^{5}$. \\ ${ }^{I}$ Department of Pharmacology, College of Pharmacy, King Saud University Riyadh Saudi Arabia; ${ }^{2}$ College of \\ Pharmacy, Princess Nourah bint Abdulrahman University, Riyadh KSA; ${ }^{3}$ Department of Genetics and Cytology; \\ National Research Center; Dokki; Egypt; ${ }^{4}$ First Year Common Deanship; King Saud University; Riyadh - KSA. \\ ${ }^{5}$ Anatomy Department, Faculty of Medicine, King Saud University, Riyadh KSA.
}

\begin{abstract}
Acetaminophen (APAP) is a widely-used analgesic, while toxic doses of which induce liver injury. Inducible cyclooxygenase-2 (COX-2) is derived prostaglandins which play an anti-inflammatory role in acetaminopheninduced hepatotoxicity. Selective activation of vascular endothelial growth factor (VEGFR1, flt -1) on endothelial cells increased mRNA levels of hepatocyte mitogens (IL-6) and hepatocyte growth factor leading to prosurvival effects on hepatocytes. The aim of this study was to compare the hepatoprotective effect of N-acetylcysteine (NAC; the antidote for APAP) with that of $\alpha$-Lipoic acid (ALA) and/or Thymoquinone (THQ) either alone or in combination on liver injury induced by APAP.

APAP administration elevated most of the previously measured parameters and decreased GSH, SOD, and total protein levels compared with the control group. Liver sections of $H \& E$ demonstrate liver injury characterized by centrilobular hepatocellular necrosis, COX-2, and flt-1 expressions were also increased. Treatment with all fore mentioned antioxidants ameliorated most of the altered parameters compared to APAP-treated group. Treatment with the combination of $A L A$ and THQ was the most effective therapy in the attenuation of liver injury assessed by a decrease in ALT and ALP activities and down-regulation of COX-2 and flt-1 expression. Section of liver from rat received APAP, ALA and THQ shows a marked improvement of hepatic degeneration which restricted to few hepatocytes with mild vacuolation of their cytoplasm while the nuclei appear normal mimic to control cells. It was concluded that the natural antioxidants such as ALA and THQ, may be considered as a potential antidote in combating liver injury induced by APAP.
\end{abstract}

Key words: Acetaminophen; $\alpha$-Lipoic acid; Thymoquinone; cyclooxygenase-2

*Author for correspondence: Hsameh2312003@yahoo.com 


\section{INTRODUCTION}

Liver damage induced by a drug is a world wide's health problem. It is a major health issue that challenges not only health care professionals but also the pharmaceutical industry and drug regulatory agencies ${ }^{1}$. Acetaminophen (N-acetyl-paminophenol, APAP; paracetamol) is commonly prescribed analgesic and antipyretic drug producing dose-dependent hepatic- and nephrotoxicity ${ }^{2}$.

The reactive metabolite of APAP is N-acetyl-p-benzoquinone imine (NAPQI), which is formed by cytochrome P-450 (CYP) by a direct two electron oxidation of $\mathrm{APAP}^{3,4}$. The reaction of NAPQI with glutathione occurs by conjugation to form 3glutathion-S-yl-acetaminophen and by reduction to acetaminophen ${ }^{5}$. Moreover, the reaction could be catalyzed by glutathione transferase, and NAPQI is one of the best substrates ever described for this enzyme ${ }^{6}$.

Treatment of mice with APAP reduced mRNA levels of TNF- $\alpha$ and other cytokines (IL-10, Il-6, COX-2, and Il-18) and there was an increase in liver toxicity assessed by increased serum ALT. Ju and colleagues postulated that these findings suggest alternative roles for Kupffer cells in the toxicity, and suggest that Kupffer cells may counteract inflammation or have a role in liver repair ${ }^{7}$.

Reilly and coworkers showed that COX-2 was induced in livers of APAP-treated mice with COX-2 playing an anti-inflammatory role in APAP-induced hepatotoxicity ${ }^{8}$.

Increased expression of vascular endothelial growth factor, VEGFR1 (flt -1), VEGFR2, and VEGFR3 protein was apparent throughout the time course of APAP administration, with peak expression during 8 to $72 \mathrm{~h}^{9}$.

Natural antioxidants are alternatives best ways to minimize liver damage and were used prophylactically and also as antidotes.

ALA is synthesized by the liver and other tissues. It is a natural cofactor in pyruvate dehydrogenase complex where it binds acyl groups and transfers them from one part of the complex to another ${ }^{10}$. ALA is a potent antioxidant. Three distinct antioxidant actions of ALA and its reduced form, dihydroLipoic acid, have been observed: reactive oxygen species scavenging activity; capacity to regenerate endogenous antioxidants such as glutathione and vitamins $\mathrm{C}$ and $\mathrm{E}$ and metal -chelating activity ${ }^{10}$.

Abdel-Zaher et al. ${ }^{11}$ reported that pretreatment of rats with ALA $(100 \mathrm{mg} / \mathrm{kg})$ protected against hepatotoxicity and nephrotoxicity induced by an acute oral toxic dose of APAP $(2.5 \mathrm{~g} / \mathrm{kg})$ as assessed by biochemical measurements and by histopathological examination.

Thymoquinone (THQ) is the most potent component of Nigella sativa. Protective effects of THQ were established in doxorubicin, carbon tetrachloride, cisplatin, ethanol and aflatoxin-induced oxidative damage. In addition, the anti-inflammatory, anti-tumoral, anti-microbial, anti-histaminic and immuno-modulatory effects of THQ have been reported. Moreover, it has been suggested that THQ may act as an antioxidant agent and prevent the membrane lipid peroxidation in hepatocytes ${ }^{12}$. So the present investigation was carried out to study the hepatoprotective effects of THQ and ALA either alone or in combination compared with that of $\mathrm{N}$ acetylcysteine (NAC) in APAP- induced toxicity model.

\section{MATERIALS AND METHODS}

\section{Experimental Animals:}

Thirty-six healthy male albino rats $(120-160 \mathrm{~g})$ were supplied by the Experimental Animal Center, College of Pharmacy, King Saud University. Animals were kept in special cages and maintained on a constant 12-h light/12-h dark cycle with air 
$\alpha$-lipoic acid and Thymoquinone protective effect

conditioning and a controlled temperature of $20-22{ }^{\circ} \mathrm{C}$ and humidity of $60 \%$. Rats were fed a standard rat pellet chow with free access to tap water ad libitum for 1 week before the experiment for acclimatization. Animal utilization protocols were performed in accordance with the guidelines provided by the Experimental Animal Laboratory and approved by the Animal Care and Use Committee of the College of Pharmacy, King Saud University.

\section{Chemicals:}

All chemicals used were of high analytical grade, a product of Sigma and Merck companies. APAP, NAC, THQ and ALA were purchased from Sigma Chemical Co. (Sigma, St. Louis, MO, USA while.

\section{Experimental design}

One week after acclimation, the rats fasted overnight before treatment and randomly divided into six groups, each of six rats as follows, Group 1: a control group which was administered saline. Group 2: rats were administered in a single oral dose of APAP $750 \mathrm{mg} / \mathrm{kg}^{13}$.

The fore mentioned antioxidants were administered in oral three doses of NAC $20 \mathrm{mg} / \mathrm{kg}^{14}$, ALA $200 \mathrm{mg} / \mathrm{kg}^{15}$ and/or THQ $15 \mathrm{mg} / \mathrm{kg}^{16}$ either alone or in combination (G3, G4 ,G5 ,G6 ), respectively. The first dose was $24 \mathrm{~h}$ before and the second was two hours after a single dose of $750 \mathrm{mg} / \mathrm{kg}$ of APAP, then a third dose of the antioxidants were taken after $12 \mathrm{~h}$ from APAP administration. One day after APAP administration, all animals were sacrificed; blood samples were withdrawn from and serum were separated by centrifugation at $3000 \mathrm{rpm}$ for $20 \mathrm{~min}$ and used for biochemical serum analysis. After blood collection, the livers were collected, washed using chilled saline solution. The livers were minced and homogenized in ice-cold bi-distilled water to yield $20 \%$ homogenates. The homogenates were centrifuged for $20 \mathrm{~min}$ at $3000 \mathrm{rpm}$ at $5^{\circ} \mathrm{C}$, and the supernatants were used for biochemical tissue analysis. Three livers from each group were kept in $4 \%$ formalin for histopathological examination and immunohistochemistry examinations.

\section{Biochemical analysis \\ Serum Biochemical Assays}

Serum Alanine Aminotransferase (ALT), Aspartate Aminotransferase (AST), Lactate dehydrogenase (LDH), Alkaline Phosphatase (ALP), total bilirubin and total protein levels were measured using commercial diagnostic kits from Randox Company (UK) following the manufacturer's instructions.

\section{Determination of lipid peroxidation (MDA) in liver tissues}

The degree of lipid peroxidation in hepatic tissues was determined by measuring thiobarbituric acid reactive substances (TBARS) in the liver homogenate ${ }^{17}$. The absorbance was measured spectrophotometrically at $532 \mathrm{~nm}$.

\section{Determination of hepatic glutathione (GSH)}

Reduced glutathione (GSH) was determined using the method of Ellman GL ${ }^{18}$ based on its reaction with 5,5'-dithiobis (2-nitrobenzoic acid) to yield the yellow chromophore, 5-thio-2-nitrobenzoic acid at $412 \mathrm{~nm}$.

Determination of hepatic total nitrite concentration 
Total nitrite was measured according to the method described Moshage et al. ${ }^{19}$ using Griess reagent (sulfanilamide and N-1-naphthylethenediamine dihydrochloride) in acidic medium.

\section{Histological Analysis}

Liver specimens were excised and stored in $4 \%$ paraformaldehyde in phosphate buffer over- night, embedded in paraffin wax at $57^{\circ} \mathrm{C}$.Serial sections were cut at 4 $\mu \mathrm{m}$ using a Spencer 820 microtome. These sections were used for Histopathological examination using $H \& E$ stains were performed.

\section{Immunohistochemistry study of COX-2 and flt}

Immunostaining of hepatic paraffin sections for detection of the abnormal immune reaction of different primary antibodies (COX-2 and flt) was performed using streptavidin-biotinylated horseradish peroxidase (S-ABC) method (Novalink Max Polymer detection system, Novocastra). The procedure involved the following steps: endogenous peroxidase activity was inhibited by $3 \% \mathrm{H}_{2} \mathrm{O}_{2}$ in distilled water for 5 minutes, and then the sections were washed in Tris-buffered saline (TBS) (Sigma, T 5030-100 TAB, PH 7.6) for 10 minutes. Non-specific binding of antibodies was blocked by incubation with protein block for 5 minutes (Novocastra). Sections were incubated with rabbit polyclonal or mouse anti-rat primary antibodies diluted 1:500 for 1 hour at room temperature. Sections were washed in Tris buffer for 3 times each for 3 minutes, then incubated with biotinylated anti-rabbit IgG (Novocastra) for 30 minutes. This was followed by washing in Tris buffer for 3times, each for 3 minutes, and then incubated with Novolink polymer (Novocastra) for 30 minutes. Then sections were washed in Tris buffer for 3 times, each for 3 minutes. Peroxidase was detected with working solution of Diaminobenzidine (DAB) substrate (Novocastra) for 10 minutes. Finally, sections were washed in distilled water for 10 minutes, nuclei were stained with Mayer's hematoxylin and sections were mounted in DPX. For negative control sections, the same procedure was followed with the omission of incubation in primary antibodies ${ }^{20}$.

\section{Statistical Analysis}

Statistical analysis was performed using GraphPad Prism (GraphPad Software, San Diego, Calif., USA), and all statistical comparisons were made by means of the oneway analysis of variance test followed by Tukey's test post hoc analysis. Results were expressed as mean $\pm \mathrm{SE}$ of the mean, and a $p$-value $\leq 0.01$ was considered significant.

\section{RESULTS}

\section{Effect of ALA \& THQ either alone or combination on APAP liver toxicity}

APAP administration exhibited a significant increase in Serum ALT, AST, LDH, ALP and total bilirubin levels ( $\mathrm{P} \leq 0.01)$ compared to normal control values (Fig. 1), with a significant decrease in serum total protein level at $P \leq 0.01$. NAC alone, ALA and/or THQ either alone or in combination successively alleviated the changes in previous mentioned serum biochemical parameters (Fig. 2).

Data represented in Fig. 1 shows the effect of APAP on oxidative stress and antioxidant biomarkers, there were an increase in NO and MDA levels and decrease SOD and GSH levels compared to control rats $(P \leq 0.01)$. Oral supplementation of NAC along with the APAP ameliorated the altered oxidative stress parameters. ALA and THQ combination treatment revealed the best results compared with NAC group or to each one alone.

\section{Histopathological changes}


$\alpha$-lipoic acid and Thymoquinone protective effect

Microscopic investigation of H\&E-stained liver sections from the control rats demonstrated normal liver histoarchitecture. Liver sections of the APAP-induced rats show multiple large foci of degenerated hepatocytes with vacuolated cytoplasm and pyknotic nuclei, it showed several widened areas of inflammation surrounding degenerated and hepatic cell necrosis.

NAC treatment markedly decreased the damage in rat liver and inflammatory cellular infiltration when compared with The APAP control rats. Liver section from rat received APAP and ALA shows also marked improvement of hepatic cells degeneration with few cells with minimal cytoplasmic degeneration, liver section from rat received APAP and THQ shows small foci of hepatic cells with moderate cellular degeneration. Section of liver from rat received APAP, ALA and THQ shows a marked improvement of hepatic degeneration which restricted to few hepatocytes with mild vacuolation of their cytoplasm while the nuclei appear normal mimic to control cells (Fig. 3A-F).

\section{Effect of ALA \& THQ either alone or combined on flt-1 and COX-2}

The immunohistochemical staining of the liver with anti flt-1 and anti COX-2 primary antibody revealed normal weak immune staining of the cytoplasm of few hepatic cells and cells in the endomysium of liver from normal control (Fig. 4\&5 A). APAP-administered rats showed the diffuse strong positive immune reaction of the nuclei of the inflammatory cells in the endomysium and the cytoplasm of degenerated liver cells (Fig. 4\&5 B). Coadministration of APAP with NAC, ALA, THQ and combined group showed a marked decrease of the immune reaction, especially the hepatic cells cytoplasm (Fig. $4 \& 5$ C-F) .

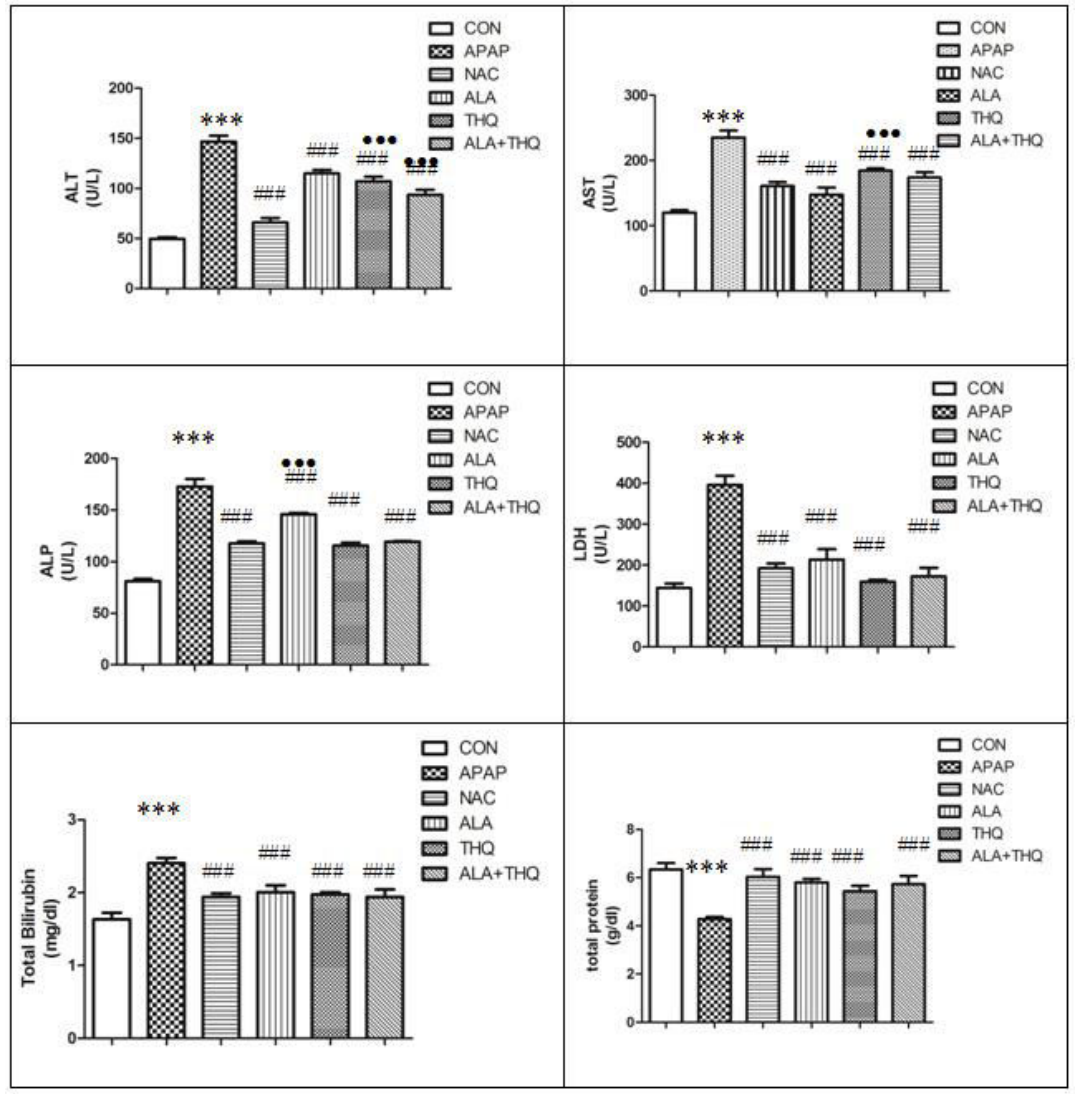

Fig. 1 Effect of NAC, ALA and THQ on serum biochemical parameters levels in APAP intoxicated rats.

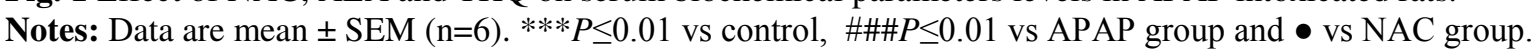




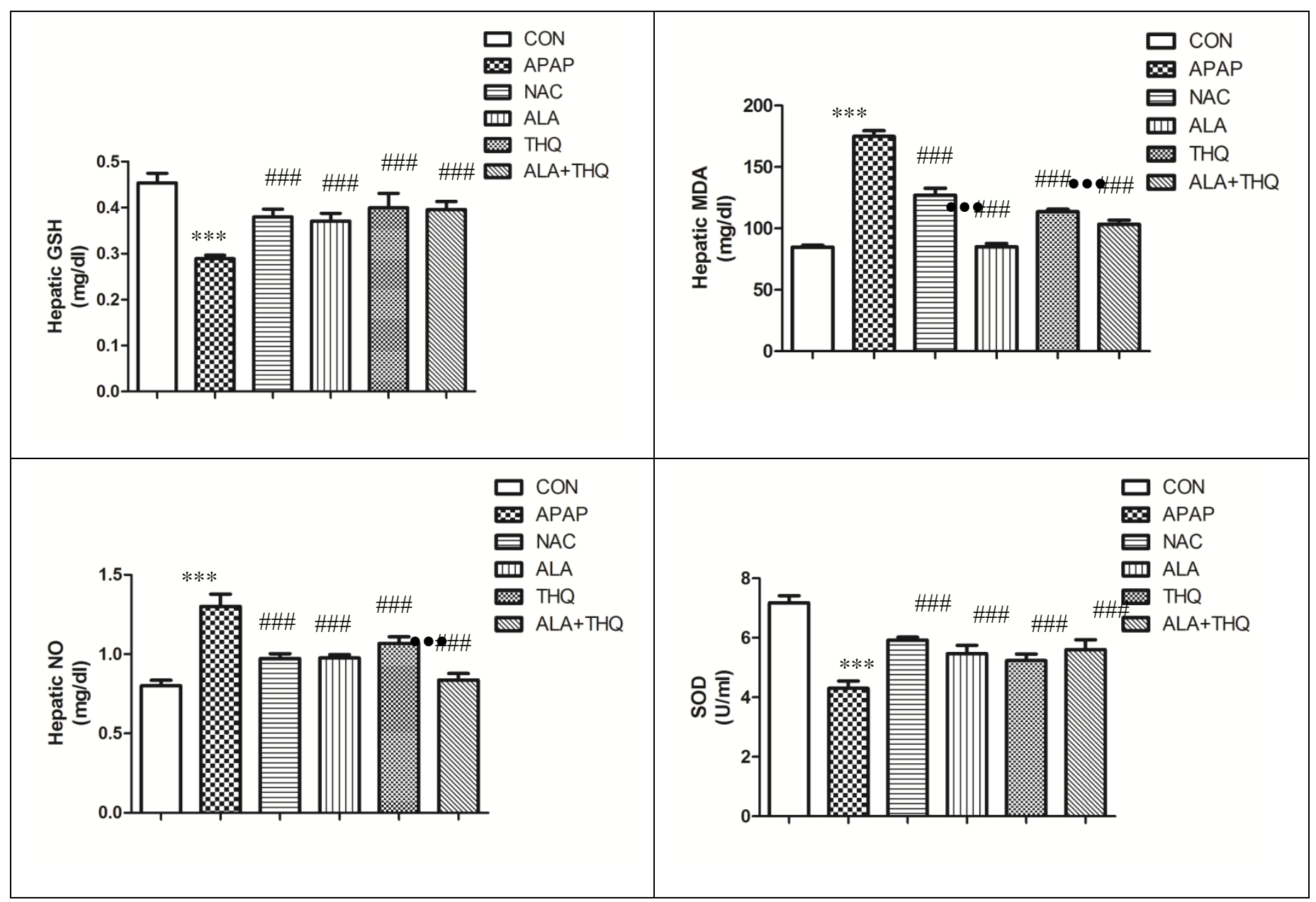

Fig. 2 Effect of NAC, ALA, and THQ on hepatic biochemical parameters levels in APAP intoxicated rats. Notes: Data are mean $\pm \operatorname{SEM}(\mathrm{n}=6) . * * * \leq 0.01$ vs control and $\# \# \# P \leq 0.01$ vs APAP group.

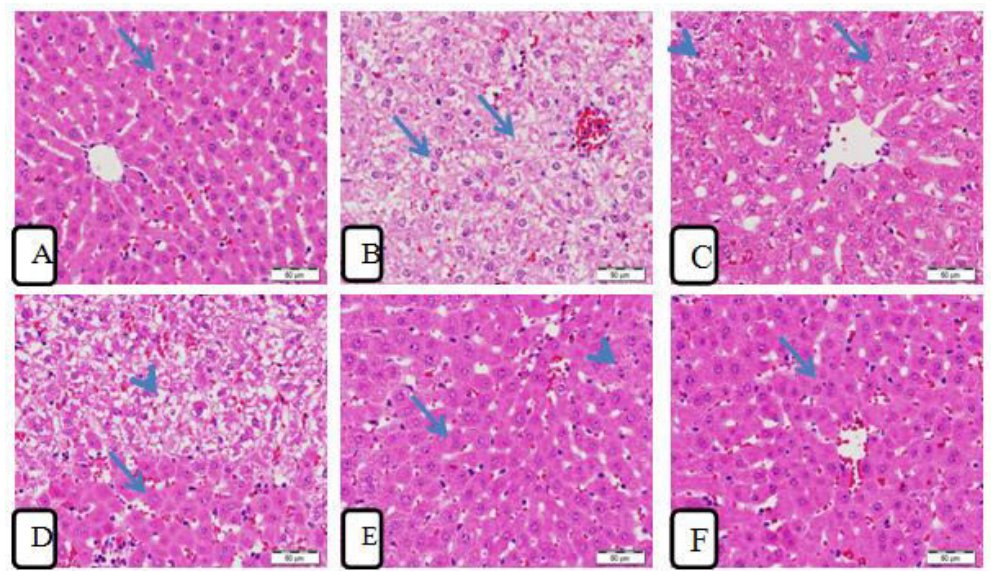

Fig. 3: light photomicrograph of a liver section stained with H\&E. Scale bar 50 $\mu \mathrm{m}$. (A) liver section from control rat showing normal hepatic architecture with normal hepatocytes (arrow) and blood sinusoids. (B) Liver section from rat received APAP shows massive cytoplasmic degeneration of the hepatocytes that appeared vacuolated (arrows) with the loss of hepatic lobular architecture. (C) Liver from rat received APAP and NAC shows a marked improvement of hepatocellular degeneration (arrow) with the restoration of normal lobular architecture. There are few mildly degenerated hepatocytes (arrowhead). (D) Liver section from rat received APAP and THQ shows also improvement of most of the degenerated hepatocytes (arrow) and lobular architecture, but still there are small areas of degenerated hepatocytes (arrowhead). (E) Liver section from rat received APAP and ALA 
$\alpha$-lipoic acid and Thymoquinone protective effect

shows a marked renewal of the degenerated hepatocytes (arrow) with few mildly degenerated cells (arrowhead). (F) Liver section from rat received APAP and combination of THQ and ALA shows normal hepatic lobular architecture and hepatocytes mimic to control section (arrow).

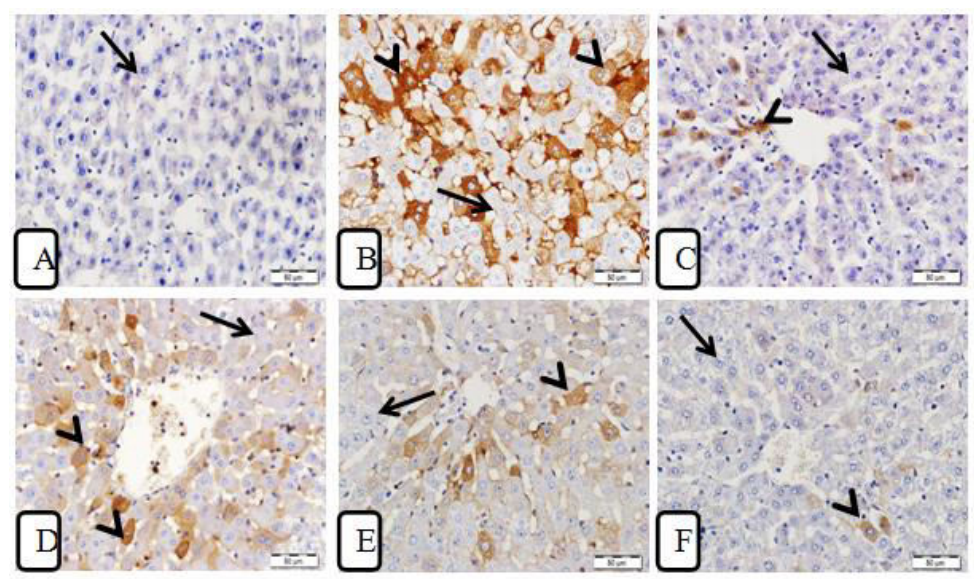

Fig. 4: light photomicrograph of liver sections Immuno-stained with the anti flt-1 primary antibody. Scale bar $50 \mu \mathrm{m}$. (A) liver section from control rat shows the normal absence of immune reactivity (arrow). (B) liver section from rat received APAP shows a marked increase of the strongly immunostained cells " cell membrane and cytoplasm' (arrowheads) between few unstained hepatocytes (arrow). (C) liver section from rat received APAP and NAC show few immuno-positive hepatocytes (arrowhead) between unstained normal cells (arrow), (D) liver section from rat received APAP and THQ shows a moderate decrease of the density and number of immunostained cells in the center-lobular zone (arrowheads) between unstained cells (arrow). (E) Liver section from rat received APAP and ALA showing many hepatocytes with immuno-negative reaction (arrow) while immune positive cells are less with weak positivity (arrowhead). (E) Represent liver section from rat received APAP and combination of THQ and ALA shows apparently normal unstained hepatocytes (arrow) with few scattered weak immuno-stained cells (arrowhead).

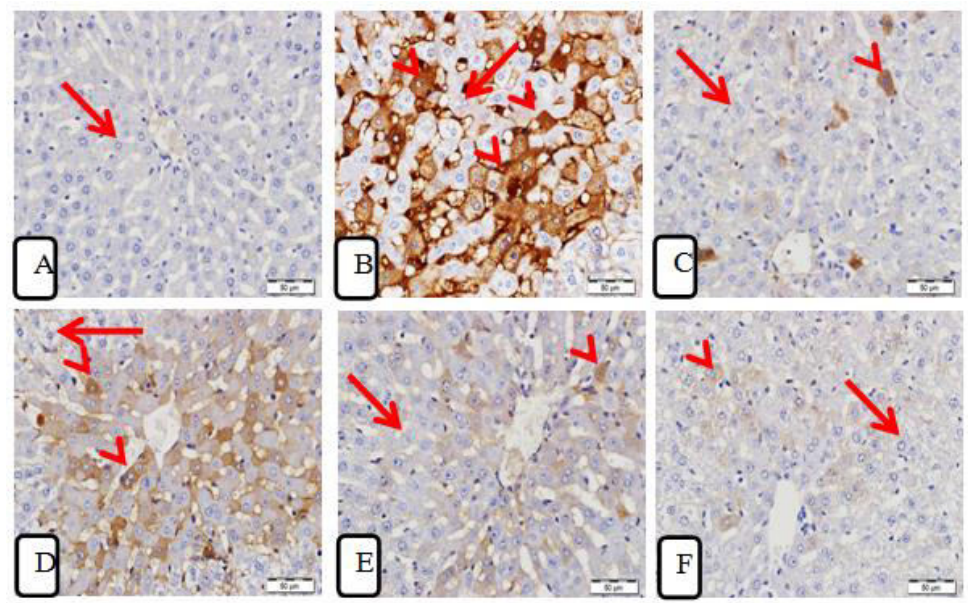

Fig. 5: light photomicrograph of liver sections Immuno-stained with the anti COX-2 primary antibody. Scale bar $50 \mu \mathrm{m}$. (A) liver section from control rat shows the normal absence of immune reactivity (arrow). (B) liver section from rat received APAP shows a marked increase of the strongly immunostained hepatocytes (arrowheads) between few unstained hepatocytes (arrow). (C) liver section from rat received APAP and NAC show few immuno-positive hepatocytes (arrowhead) between many unstained normal cells (arrow), (D) liver section from rat received APAP and THQ shows a moderate decrease of the density and number of immunostained cells in the center-lobular zone (arrowheads) between unstained cells (arrow). (E) Liver section from rat received APAP and ALA shows many hepatocytes with immuno-negative reaction (arrow) while immune positive cells are less with weak positivity (arrowhead). (E) Represent liver section from rat received APAP and combination of THQ and ALA shows apparently normal unstained hepatocytes (arrow) with few scattered very weak immuno-stained cells (arrowhead). 


\section{DISCUSSION}

Although APAP is considered safe at therapeutic doses, at higher doses, APAP produces a centrilobular hepatic necrosis that can be fatal. Today APAP poisoning accounts for approximately one-half of all cases of acute liver failure in the United States and Great Britain ${ }^{21,22}$. APAP hepatotoxicity occurs by a complex mechanistic sequence. The reactive metabolite NAPQI which depletes glutathione by a conjugation reaction and covalently binds to proteins; loss of glutathione causes an increased oxidative stress response (decreased detoxification of reactive oxygen and nitrogen species); increased oxidative stress, possibly associated with alterations in calcium metabolism ${ }^{23}$. Then initiation of signal transduction responses and mitochondrial permeability transition; which occurs with an even larger increase in oxidative stress, loss of mitochondrial membrane potential, however, the loss of ability of the hepatocyte mitochondria to produce ATP may be the most important event causing necrosis ${ }^{24}$. In addition, there are a number of modulators of inflammatory responses that can alter the severity of liver injury following the initiation of toxicity ${ }^{25,26}$. The interactions of these mediators with each other and the interplay of the immune cells that produce them will help to elucidate the significance of their roles in APAP toxicity ${ }^{23}$. Apoptotic responses occur in conjunction with these inflammatory events ${ }^{27,} 28$. Finally, liver has a very great capacity to regenerate and various cytokines and growth factors are the major initiators of this process.

Gardner et al. ${ }^{29}$ reported that toxic doses of APAP to rats induced hepatic-inducible nitric oxide synthase (iNOS) in the centrilobular hepatocytes. The development of toxicity is correlated with the expression of iNOS.

APAP-administration induced profound elevation of nitric oxide (NO) production and oxidative stress, as evidenced by increasing of lipid peroxidation level, reducing of SOD activity and depleting of intracellular GSH level in liver and kidney ${ }^{11}$.

Moreover Prescott ${ }^{30}$ reported that a dramatic increase in serum ALT and AST levels, mild hyperbilirubinemia, and increased prothrombin time are resulted from the biochemical changes after APAP administration. Moreover, the formation of superoxide and nitric oxide that react together to produce peroxynitrite, which again exhibits hydroxyl radical-like activity causing hepatotoxicity which is contributed by hepatic macrophages via different mechanisms ${ }^{31}$. These results are in accordance with those of the current work as APAP administration induced an elevation of liver biomarker enzymes while treatment with NAC and ALA and/or THQ either alone or in combination attenuate all the altered parameters. Our results are documented by those of Abdel-Zaher et al. ${ }^{11}$ who reported that ALA downregulated the elevation of NO and oxidative stress, as evidenced by decreasing of lipid peroxidation level, reducing of glutathione peroxidase (GSH-Px) activity and increasing the depletion of intracellular GSH level in liver and kidney. These results provide evidence that the inhibition of $\mathrm{NO}$ overproduction and maintenance of intracellular antioxidant status may play a pivotal role in the protective effects of ALA against APAPinduced hepatic and renal damage. Moreover, our results are in accordance with that of Aycan et al. ${ }^{12}$ who reported that co-administration of APAP with THQ serum ALT, AST levels were increased, whereas serum and tissue MDA levels were found to be lower compared to that of the APAP treated group. Histopathological analysis revealed a significant liver necrosis and toxicity with a high dose of APAP where THQ treatment significantly lowered liver injury scores. 
$\alpha$-lipoic acid and Thymoquinone protective effect

Another anti-inflammatory mechanism that has been reported to play a role in APAP toxicity is cyclooxygenase- $2(\mathrm{COX}-2)^{8}$. COX-2 is inducible and plays a role in antiinflammatory processes. COX-2 generates prostaglandins are recognized for their critical role in female reproduction, bone resorption, renal function, and mucosal defense. Prostaglandins have been reported to play a protective role in various hepatotoxicities ${ }^{32}$. Reilly and coworkers ${ }^{8}$ showed that COX-2 was induced in livers of APAP-treated mice; it plays an anti-inflammatory role in APAP-induced hepatotoxicity ${ }^{8}$. In the present work immune stain with anti COX-2 revealed that APAP exhibits strong immune response while treatment with NAC and ALA and/or THQ either alone or in combination downregulates COX-2 expression. Mansour ${ }^{33}$ reported that inflammation is also mediated by enzyme cyclooxygenase (COX) enzyme COX generates prostaglandins (PGEs) and thromboxane from arachidonic acid. Therefore, both PGEs and LTs function as the main mediators of allergy and inflammation. THQ inhibited both COX and lipooxygenase (LO) pathways of arachidonate metabolism in rats. Thus, inhibition of both COX and LO pathways is a key factor mediating the anti-inflammatory effects of THQ. Also Hegazy ${ }^{34}$ supported our work and reported that ALA was effective in minimizing age-related oxidative burden. ALA reduced the levels of the pro-inflammatory cytokines IL-1, IL-6, and TNF- $\alpha$, in addition to inhibiting the activity of COX-2.

VEGF induces the expression of anti-apoptotic proteins in human endothelial cells, suggesting that it promotes the survival of these cells. VEGF mRNA transcripts and protein are expressed by other cell types as well, including hepatocytes ${ }^{35}$. VEGF has two primary receptors, VEGFR1 (flt-1) and VEGFR2. Data suggest that VEGR1 may have probiotic effects. Activation of VEGFR1 in vitro resulted in significant increases in the release of mitogens by endothelial cells ${ }^{36}$. These mitogens included the known hepatocyte mitogens: hepatocyte growth factor (HGF) and IL-6. Hepatic VEGF1 (flt-1) level was increased 30-fold in mice treated with APAP and followed the onset of toxicity ${ }^{9}$. Moreover, treatment with ALA inhibits VEGF expression by blocking the superoxide anion, causing a decrease in vascular leakage and tissue edema reduction. Taken together, edema formation is the result of synergism between various inflammatory mediators that increase vascular permeability and/or the mediators that increase blood flow ${ }^{37}$.

Paarakh $^{38}$ reported that THQ downregulate the expression of VEGF1. The present results revealed that administration of NAC and ALA and/or THQ either alone or in combination along with APAP downregulates flt-1 expression.

Further studies are needed to examine the effect of inflammatory mediators on oxidative stress and/or signal transduction responses in APAP toxicity. Also, we can conclude that the administration of a combination of ALA and THQ is considered as a safe antidote for APAP toxicity.

\section{CONCLUSION}

It was concluded that the natural antioxidants such as ALA and THQ, may be considered as a potential antidote in combating liver injury induced by APAP.

\section{ACKNOWLEDGMENT}

This research project was supported by a grant from the 'Research Center of the Center for Female Scientific and Medical Colleges', Deanship of Scientific Research, King Saud University. 


\section{REFERENCES}

1. Saleem MT, Christina AJ, Chidambaranathan N, Ravi V, Gauthaman K. Hepatoprotective activity of Annona squamosa (Linn) on experimental animal model. International Journal of Applied Research in Natural Processes. 2008;1(3): 1 - 7.

2. James LP, Mayeux PR, Hinson JA. Acetaminophen-induced hepatotoxicity. Drug Metab Dispos. 2003; 31:1499-1506.

3. Dahlin DC, Miwa GT, Lu AY, Nelson SD. N-acetyl-p-benzoquinone imine: a cytochrome P-450-mediated oxidation product of acetaminophen. Proc Natl Acad Sci USA. 1984;81,1327-1331.

4. Potter DW, Hinson JA. Mechanisms of acetaminophen oxidation to N-acetyl-Pbenzoquinone imine by horseradish peroxidase and cytochrome P-450. J Biol Chem. 1987;262,966-973.

5. Dahlin DC, Nelson SD. Synthesis, decomposition kinetics, and preliminary toxicological studies of pure N-acetyl-p-benzoquinone imine, a proposed toxic metabolite of acetaminophen. J Medicin Chem. 1982;25(8), 885-886.

6. Coles B, Wilson I, Wardman P, Hinson JA, Nelson SD, Ketterer B. The spontaneous and enzymatic reaction of $\mathrm{N}$-acetyl-p-benzoquinonimine with glutathione: a stopped-flow kinetic study. Arch Biochem Biophys. 1988;264:253-260.

7. Ju C, Reilly TP, Bourdi M, Radonovich MF, Brady JN, George JW, et al. Protective role of Kupffer cells in acetaminophen-induced hepatic injury in mice. Chem Res Toxicol. 2002;15,1504-1513.

8. Reilly TP, Brady JN, Marchick MR, Bourdi M, George JW, Radonovich MF, et al. A protective role for cyclooxygenase-2 in drug-induced liver injury in mice. Chem Res Toxicol. 2001; 14,1620-1628.

9. Donahower B, McCullough SS, Kurten R, Lamps LW, Simpson P, Hinson JA, et al. Vascular endothelial growth factor and hepatocyte regeneration in acetaminophen toxicity. Am J Physiol Gastrointest Liver Physiol. 2006; 291,G102-G109.

10. Biewenga GP, Haenen GR, Bast A. The pharmacology of the antioxidant Lipoic acid. Gen Pharmacol. 1997; 29, 315-331.10.1016/S0306-3623(96)00474-0

11. Abdel-Zaher AO, Abdel-Hady RH, Mahmoud MM, Farrag MM. The potential protective role of alpha-Lipoic acid against acetaminophen-induced hepatic and renal damage. Toxicol. 2008; 243(3),261-70.

12. Aycan IÖ, Tüfek A, Tokgöz O, Evliyaoğlu O, Frrat U, Kavak GÖ, et al. Thymoquinone treatment against acetaminophen-induced hepatotoxicity in rats. Int $J$ Surg. 2014;12(3),213-8.

13. Zhao C, Shichi H. Prevention of acetaminophen-induced cataract by a combination of diallyl disulfide and N-acetylcysteine. J Ocul Pharmacol Ther. 1998;14(4),345-55.

14. Pinho RA, Silveira PC, Silva LA, Luiz SE, Dal-Pizzol FF, Moreira JC. N-acetylcysteine and deferoxamine reduce pulmonary oxidative stress and inflammation in rats after coal dust exposure. Environ Res. 2005; 99(3),355-360.

15. Yang Y, Lib W, Liub Y, Sunb Y, Lib Y, Yaob Q, et al. Alpha-Lipoic acid improves high-fat diet-induced hepatic steatosis by modulating the transcription factors SREBP-1, FoxO1 and Nrf2 via the SIRT1/LKB1/AMPK pathway. J Nut Biochem. 2014; 25(11), 1207-1217.

16. Abukhader MM. The effect of route of administration in thymoquinone toxicity in male and female rats. Indian J Pharm Sci. 2012;74(3),195-200. doi: 10.4103/0250474X.106060.

17. Uchiyama M, Mihara M. Determination of malondialdehyde precursor in tissues by thiobarbituric acid teat. Anal Biochem. 1978;86, 271-278.

18. Ellman GL. Tissue sulfhydryl groups. Arch Bioachm Biophys. 1959;82, 70-74.

19. Moshage H, Kok B, Huizenga JR, Jansen PL. Nitrite and nitrate determination in plasma: a critical evaluation. Clin Chem. 1995;41,892-896. 
$\alpha$-lipoic acid and Thymoquinone protective effect

20. Al-Rasheed NM, Attia HA, Mohamad RA, Al-Rasheed NM, Al-Amin M, AL-Onazi A. Aqueous Date Flesh or Pits Extract Attenuates Liver Fibrosis via Suppression of Hepatic Stellate Cell Activation and Reduction of Inflammatory Cytokines, Transforming Growth Factor- $\beta 1$ and Angiogenic Markers in Carbon Tetrachloride-Intoxicated Rats. Evid Based Complement Alternat Med. 2015;2015, 19 pages.

21. Larson AM, Polson J, Fontana RJ, Davern TJ, Lalani E, Hynan LS, et al. Acetaminopheninduced acute liver failure: results of a United States multicenter, prospective study. Hepatol. 2005;42,1364-1372.

22. Ostapowicz G, Fontana RJ, Schiodt FV, Larson A, Davern TJ, Han SH, et al. Results of a prospective study of acute liver failure at 17 tertiary care centers in the United States. Ann Intern Med. 2002; 137,947-954.

23. Hinson JA, Roberts DW, James LP. Mechanisms of acetaminophen-induced liver necrosis. Handb Exp Pharmacol. 2010;196,369-405.

24. Myhre O, Andersen JM, Aarnes H, Fonnum F. Evaluation of the probes 2', 7'dichlorofluorescin diacetate, luminol, and lucigenin as indicators of reactive species formation. Biochem Pharmacol. 2003;65,1575-1582.

25. Laskin DL, Pilaro AM, Ji S. Potential role of activated macrophages in acetaminophen hepatotoxicity. II. Mechanism of macrophage accumulation and activation. Toxicol Appl Pharmacol. 1986;86:216-226.

26. Laskin DL, Pilaro AM. Potential role of activated macrophages in acetaminophen hepatotoxicity. I. Isolation and characterization of activated macrophages from rat liver. Toxicol Appl Pharmacol 1986;86:204-215.

27. Adams ML, Pierce RH, Vail ME, White CC, Tonge RP, Kavanagh TJ, et al. Enhanced acetaminophen hepatotoxicity in transgenic mice overexpressing BCL-2. Mol Pharmacol. 2001;60,907-915. [PubMed]

28. El-Hassan H, Anwar K, Macanas-Pirard P, Crabtree M, Chow SC, Johnson VL, et al. Involvement of mitochondria in acetaminophen-induced apoptosis and hepatic injury: roles of cytochrome c, Bax, Bid, and caspases. Toxicol Appl Pharmacol. 2003;191:118129.

29. Gardner CR, Heck DE, Yang CS, Thomas PE, Zhang XJ, DeGeorge GL, et al. Role of nitric oxide in acetaminophen-induced hepatotoxicity in the rat. Hepatol.1998; 27, 748754.

30. Prescott LF. Therapeutic misadventure with paracetamol: fact or fiction? Am J Ther. 2000; 7,99-114.

31. Michael SL, Pumford NR, Mayeux PR, Niesman MR, Hinson JA. Pretreatment of mice with macrophage inactivators decreases acetaminophen hepatotoxicity and the formation of reactive oxygen and nitrogen species. Hepatol. 1999;30,186-195.

32. Quiroga J, Prieto J. Liver cytoprotection by prostaglandins. Pharmacol Ther. 1993;58,67-91.

33. Mansour M, Tornhamre S. Inhibition of 5lipoxygenase and leukotriene C4 synthase in human blood cells by thymoquinone. J Enzyme Inhib Med Chem. 2004;19,431-436.

34. Hegazy HG. Ameliorative effects of ginger and -Lipoic acid on oxidative stress and inflammation in senile female rats, African J Pharmacy Pharmacol. 2011;5(8), 10961105 .

35. Mochida S, Ishikawa K, Inao M, Shibuya M, Fujiwara K. Increased expressions of vascular endothelial growth factor and its receptors, Flt-1 and KDR/flk-1, in regenerating rat liver. Biochem Biophys Res Commun. 1996; 226,176-179.

36. Le Couter J, Moritz DR, Li B, Phillips GL, Liang XH, Gerber HP, et al. Angiogenesisindependent endothelial protection of liver: role of VEGFR-1. Science. 2003;299,890893.

37. Goraca A, Huk-Kolega H, Kowalczyk A, Skibska B. Anti-oxidative and antiinflammatory effects of Lipoic acid in rat liver. Postepy Hig Med Dosw (Online). 2015;69,270-6. doi: 10.5604/17322693.1142473.

38. Paarakh PM. Nigella Sativa Linn-Acomprehensive review. Indian j of natural product resources. 2010;1,409-429. 


\section{Erratum}

In Article "Hepatoprotective Role of $\alpha$-Lipoic acid and Thymoquinone in AcetaminophenInduced Liver Injury: Down-Regulation of COX-2 and flt-1 Expression", with the number of DOI: http://dx.doi.org/10.1590/1678-4324-2017160703, published in journal Brazilian Archives of Biology and Technology, vol. 60, the 01 page.

That read:

"http://dx.doi.org/10.190/1678-4324-2017160703"

Read:

"http://dx.doi.org/10.1590/1678-4324-2017160703" 